\title{
Balanced : An Application To Improve Mental Health
}

\author{
Mrunal Bhalerao ${ }^{1, *}$, Trisha Lewis ${ }^{1}$, Raj Salvi ${ }^{1}$, and Rakhi Kalantri ${ }^{2}$ \\ ${ }^{1}$ UG students, Computer Engineering Department, Fr. C. Rodrigues Institute of Technology, Navi Mumbai 400703, MH \\ ${ }^{2}$ Assistant Professor, Computer Engineering Department, Fr. C. Rodrigues Institute of Technology, Navi Mumbai 400703, MH
}

\begin{abstract}
Mental health reflects a person's emotional, psychological, and social well-being. A report by World Health Organization suggests that India is the most depressed country in the world. Hence, the idea is to develop an end-to-end solution for identifying and treating mentalhealth issues. This paper aims to develop an application to keep track of user's behaviour, both online and offline, to understand and identify the possible mental health issues using various analytical and psychological methodologies. The target users of this application would be the individuals just above the age of 18 which fall under the category of working class. Postidentification, the user is recommended a suitable change in his routine and behavior which would lead to a better lifestyle.Mental illnesses detection is a challenging undertaking because a misdiagnosis can lead to catastrophic consequences. Hence, our android application will take the necessary steps to accurately identify and treat the mental health issue.
\end{abstract}

\section{INTRODUCTION}

In [1] Mental Illness is expounded as a mental, emotional, or behavioural disorder. The severity of the impact varies from no impairment to severe impairment, which significantly hampers or interferes with one or more important living activities. Thus, proper assessment of mental wellbeing is highly essential to understand and suggest therapies to individuals who are facing mental illness. While study suggests that 1 in every 4 adults is affected by mental health problems. However, recognising mental health concerns is crucial, as misinterpretation can result in life-threatening consequences. As a result, proper attention must be made to identify mental health disorders. Additionally, only 10 to 12 percent of those who are affected seek care. Hence, the absolute necessity of today is to create awareness about mental illness and normalize the concept of seeking help to overcome this dark phase [2].

Therefore, in this paper, we intend to develop an end-to-end solution for identifying the possibility of a mental illness, as well as, provide our user with a platform to deal with his/her condition and live a better life every day. We have developed an android application and a website to keep track of user's digital, physical and psychological activities, both online and offline. These activities are then perfectly analyzed by the Machine Learning model to predict the possibility of mental disorder. Post-identification, the user is recommended a suitable change his routine and behavior which would lead to a better lifestyle. Depending on the identification, if required, the user may also be connected to a psychologist, a yoga institute, gym etc., which may help him improve his mental health condition. In addition to this, weekly and monthly evaluations will be conducted for the users where they will be presented with a brief description of their habits

*Corresponding author: mrunal.bhalerao25@gmail.com and mental-health condition in an understandable visual format. Further lifestyle changes that they can undertake to improve upon their condition will be suggested.

\section{LITERATURE REVIEW}

In [3] U. S. Reddy et. al. has applied Logistical Regression, Random Forest and Decision Tree algorithms to determine which of these is the most accurate. A number of parameters in the dataset were compared to see how they related to one another.

In [4] M. P. Dooshima et. al. has applied Decision Trees and Naïve Bayes algorithm. Demographic, psychological, biological and environmental factors are used for prediction. To validate the obtained parameters, different health professionals were approached.. However, the Data is only for 30 patients.

In [5] M. Srividya et. al. made use of a questionnaire to obtain values for different attributes which could help in prediction of mental illness. This paper intended to analyse various algorithms and determine the accurate one. She used the labels from the collected data to compute a MOS. She then applied Decision Tree, Naïve Bayes and SVM algorithms to find the more accurate. In Conclusion, Support Vector Machine and K- Nearest Neighbors algorithms have similar accuracy results and show highest accuracy.

In [5] M. Srividya et. al. used Neural Networks to determine the psychological conditions of humans such as depression, stress, anxiety etc. 


\subsection{Data Mining algorithms for Privacy- Preserving:}

\subsubsection{Randomization}

During Vertival Shuffling of Randomization records the attribute's semantic does not changes. Instead, the vertical position of the record is altered, concealing the true identity. To disguise the behaviour of individual records, noise can be added to the data. The aggregate behaviour of the data distribution is later rebuilt by removing the noise from the data[6].

\subsection{2 k-anonymity}

Data anonymization is used when it is necessary to make changes to the original data in order to remove any information that is directly traceable to any of the individuals. Here, the granularity of data representation is decreased by using suppression and generalization techniques [7].

\subsubsection{I-diversity}

The 1-diversity model overcomes the foibles of the kanonymity model. Thus, preserving identities at the level of $\mathrm{k}$-individuals differs from protecting the corresponding values, particularly when sensitive values within a group are homogeneous. The anonymization scheme encourages intra-group variation in sensitive features [10].

For Chatbot, in [9], "A Mental Health Chatbot for Regulating Emotions (SERMO) - Concept and Usability Test", mentions a mobile application integrated with a chatbot which developed using the Syn.Bot framework. It contains OSCOVA and Synthetic Intelligence Markup Language interpreter. It employs cognitive behaviour therapy (CBT) techniques to assist mentally ill individuals in regulating their emotions and dealing with their thoughts and feelings. Daily, it inquires of the user about events in their life and their emotions. And, using NLP and a lexicon-based technique, the app detects a user's core emotion from natural language input. A suitable measurement, such as activities or mindfulness exercises, is also advised.

In [11], we understand that mental health problems can be found at different ages. While, each category shows different signs, some show similar signs too. According to the paper, Mental illness can be categorized into 7 types: Personality illness, Compulsion illness, Eating illness, Emotional illness, selfish control illness, Nervousness Illness and Psychological illness.

In [8], "Data Science in Public Mental Health: A New Analytic Framework", advanced methodologies were used to conduct a thorough investigation into public mental health issues using data science and to find remedies based on the findings of the data science publications. A complete comparison of those with conventional data analysis techniques is also presented. The study proposes a new framework for mental health professionals to tackle problems and challenges they face using data science. In order to get a good concept of the problem to be solved, the research also looks into predicting the time and resources required in any process.

In [12] The research "Depression detection using emotion artificial intelligence," looks at depression as a significant cause of mental illness as well as an increased risk of dying young. Furthermore, depression is identified as a primary cause of suicide ideation, resulting in severe impairment in daily life, according to the study. It delves into emotion artificial intelligence as a subject of continuing research in emotion recognition, with a focus on text mining. They have applied NLP on Twitter feeds for demonstrating emotion analysis to focus mainly on depression. To detect depression tendencies, tweets are categorised as neutral or negative based on a chosen word list. Support vector machines and Naive-Bayes classifiers were employed in the class prediction process. The findings are determined using the F1-score, accuracy, and confusion matrix.

\section{PROPOSED SYSTEM}

Based on the literature, we propose a system whose major purpose is to create a website and an Android app, where the users will have to login through their google accounts and fill in a registration form. The form will allow us to have background information of the user. Once the user logs in, data including digital usage data, data containing mood and activities performed throughout the day, data from conversations with chatbot, Psychological health data and physical health data will be collected by the application and stored in the firebase. The data gathered will be pre-processed and analyzed. Based on this data, results about potential or possibility of mental illness will be available to the user. We will label and encode the data for better prediction. We plan on using Random Forest algorithm with Clustering for predicting a pattern within the data.

Once, a prediction is made the user will be presented with a proper treatment by connecting him/her with a psychologist, a yoga institute, a gym, etc. that may help ameliorate his mental health conditions. The user will also be suggested some to change his routine and suggest some behavioral changes which might help him improve his condition. In addition to this, we will provide in-app features including a Wellness Center, Habit Tracker, Scheduler, Music Player, connect with peers and Connect with professionals. Finally, weekly evaluations will be done and real time reports of the user's digital, physical and psychological behavior will be developed in layman a visual format. 
In this way, the solution will be an end-to-end solution for predicting the possibility of mental illness and also helping to lower the impact of mental illness.

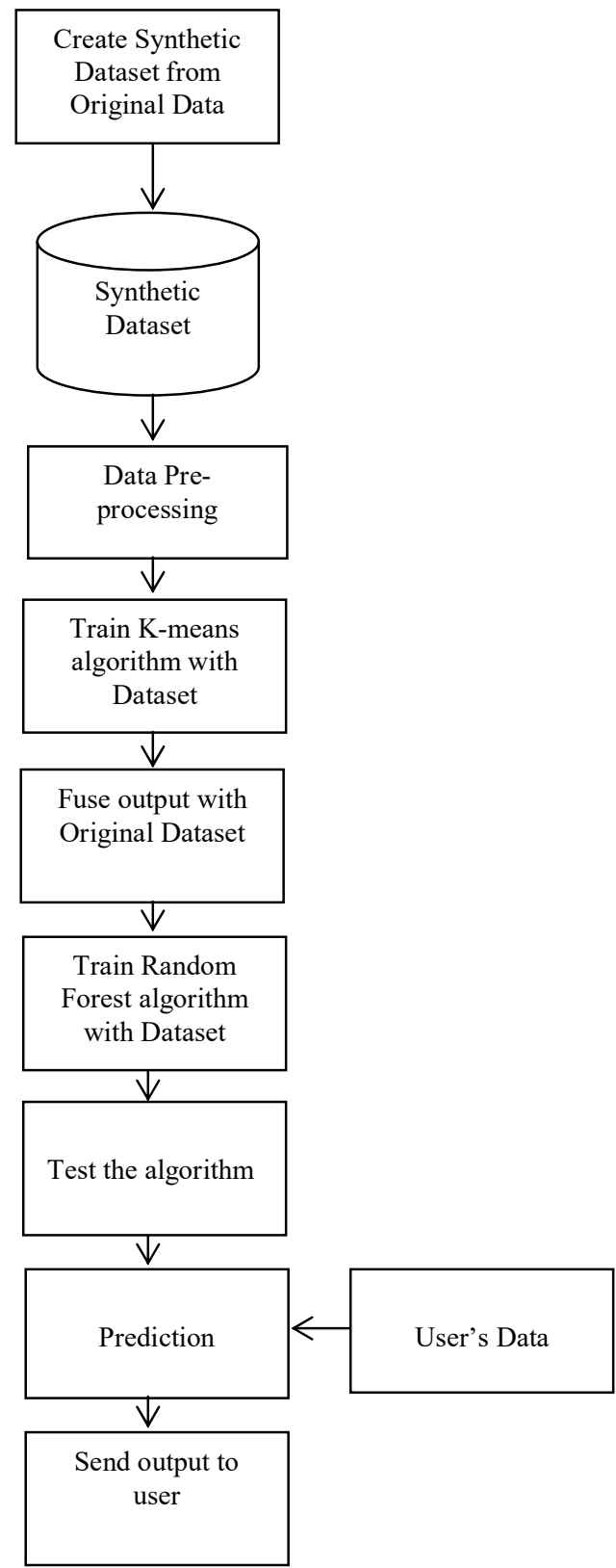

Fig. 1. System Block diagram

\section{IMPLEMENTATION AND RESULT}

\subsection{Data Collection}

The user will have to use the android/web application to login through their google accounts and fill in a registration form. The form will allow us to have background information of the user. Once the user logs in, data including:

\subsubsection{Digital Information Data}

Location, websites visited, battery levels, media playback time, applications used and duration of usage, data usage, network information, gyroscope information, accelerometer information, screen on time will be gathered and saved in the firebase.

\subsubsection{Physical Information Data:}

Number of steps walked, duration and schedule of sleep, the user will be prompted to mark a response for activities done throughout the day.

\subsubsection{Psychological Information Data:}

Responses to 4 psychometric quizzes suggested by a psychiatrist will be gathered, user will be prompted to mark a response for "how are you feeling?" multiple times a day, as shown in [9] conversations with the chatbot will be monitored and a basic emotion will be determined for every conversation.

\subsection{Data Flow/Storage:}

Data from the Web Extension will be stored on the Server (Heroku) and then on the Firestore. Data from the android application will be stored in the Firebase. In case of any discrepancy in data, it will be sent to a local server, pre-processed, and then sent to the database.

This data collected is used for prediction of possibility of mental illness. The visualization of collected data is shown to the user in a graphical format. This allows the user to verify and track changes in his behavior, which in turn satisfies are aim of evidence-based prediction. Figure 2 illustrates how real times graphs will be plotted. Figure 3 and Figure 4 are examples of plotted graphs.

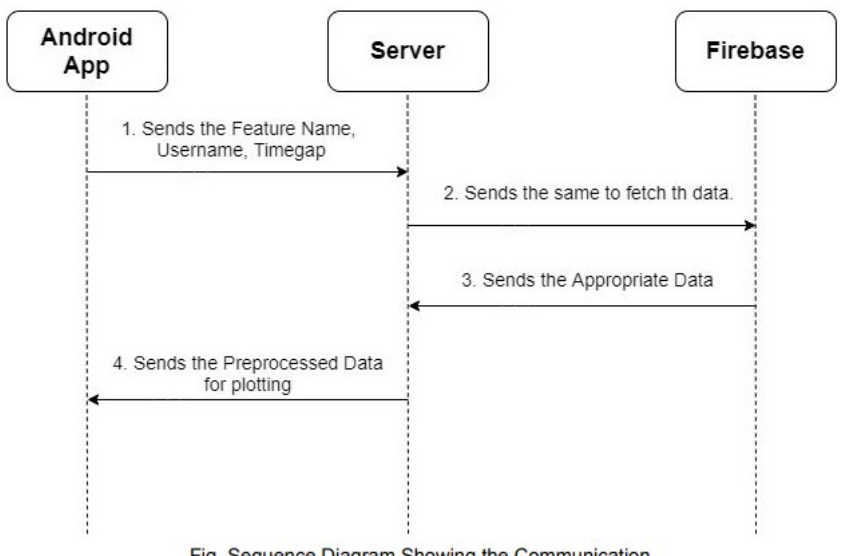

Fig. Sequence Diagram Showing the Communication

Fig. 2. Sequence Diagram 

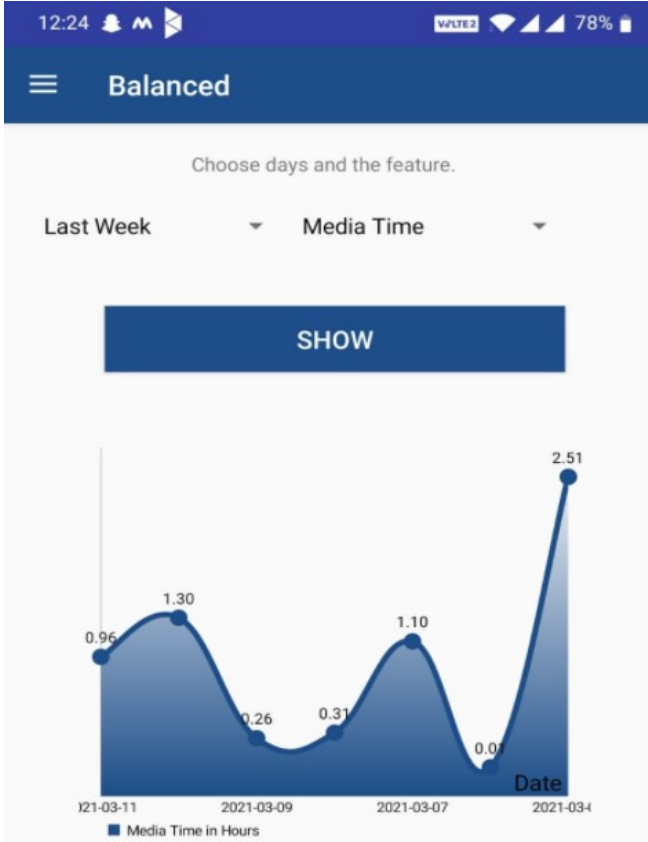

Average Media Time this Week: 0.9221825 Hours

Fig. 3. Media Time
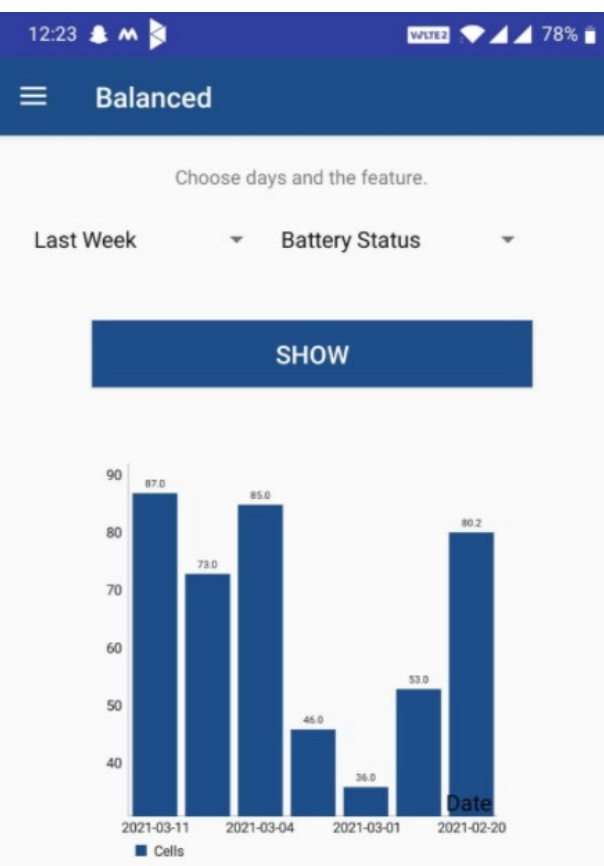

Average Battery this Week: $65.74286 \%$

\section{DETECTION}

We will forecast the user's mental ailment during the detecting phase. Some of the characteristics we are using are age, gender, family history of mental illness, results from 4 quizzes, their basic mood which is derived from the "how are you feeling?" and conversations with the chatbot, phone usage, applications used, etc. For the detection of possibility of mental illness, a synthetic dataset was formed by using the original dataset and some threshold values. The threshold values were carefully found out under guidance of a psychiatrist. Permutation and combination techniques were used to form the synthetic dataset from the original dataset.

Further, K-means clustering technique was employed to cluster the dataset into two classes: Possibility of Mental Sickness and No signs of Mental sickness. K-means clustering algorithm groups/clusters data having similar features by using the technique of Euclidian Distance. It also ranks the importance of each feature. In this scenario, quiz 3, quiz 4 and apps used are given highest priority as shown in the Table 1 .

\begin{tabular}{|l|l|}
\hline FEATURE & IMPORTANCE VALUE \\
\hline Age & 0.00198102 \\
\hline $\begin{array}{l}\text { Emotional Quotient } \\
\text { Test }\end{array}$ & 0.00179368 \\
\hline Depression Test & 0.00219795 \\
\hline Locus of control Test & $\mathbf{0 . 5 0 7 5 9 1 7 5}$ \\
\hline Optimism Test & 0.47584557 \\
\hline Screen on Time & 0.00284181 \\
\hline Social Media & 0.00250919 \\
\hline Youtube & 0.0027524 \\
\hline Others & 0.00248662 \\
\hline
\end{tabular}

Table. 1. Ranking of Features

Now, the output of the clustering was fused with the original dataset. Thus, converting the un-supervised problem to a supervised task. This data was then fed into the Random Forest algorithm (RFT) for supervised learning. RFT is a combinations of multiple decision trees. Each individual tree determines a class prediction and the class with the most votes becomes the model's final prediction of whether the user is suffering from any Mental Illness or not.

Fig. 4. Battery Status 


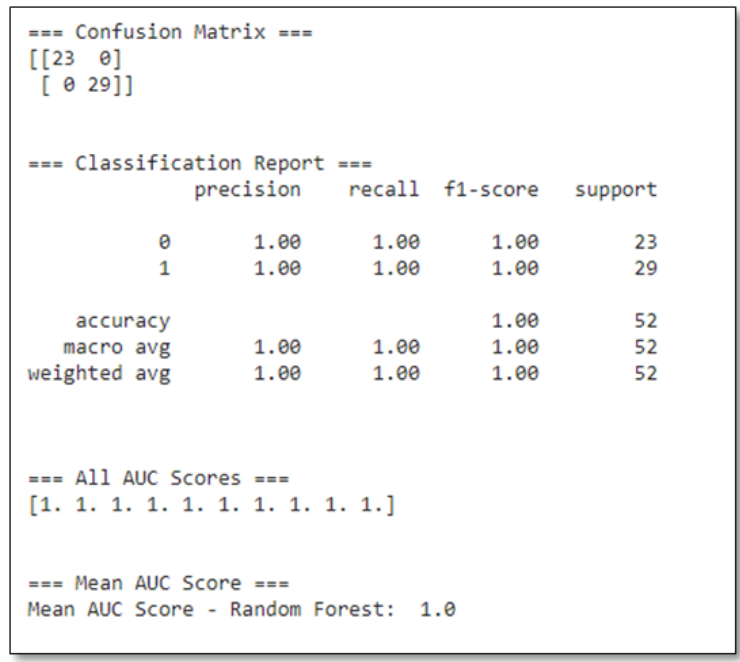

Fig. 5. Classification Report

Now, that the ML model is trained to predict the proper output, it was ready for deployment. We deployed our model on Heroku by linking the GitHub repository to the Heroku account. Heroku is nothing but a Platform as a Service. In other words, it allows to transfer the application from a local machine to the cloud. This, allows the deployment of a machine learning model in real-time.

\subsection{Aid for a Better Mental Health}

With a vision of providing guidance to improve our user's mental health condition, we have provided the following in-app features:

\subsubsection{Digital Information Data}

The Wellness Center mainly consists of the -

Virtual Gym: It consists of a carefully curated list of workouts which when performed boosts endorphin levels. They are classified on the basis of time.

Meditation Center: It consists of resources which can help the user deal with anxiety, depression, high stress levels, insomnia and anger. It will help to develop a overall better lifestyle with a positive attitude.

Learning Center: It has resources which able the user to utilize his/her time by learning something new or pursuing a hobby. This will allow the user to develop a habit of utilizing free time instead of wasting it in unproductive/disturbing activities which lead to mental sickness.

Peer Connect: This feature will allow the user to connect with other people who are willing to start a conversation. Thus, it will allow the user to socialize, which plays a vital role in boosting positive mental health.

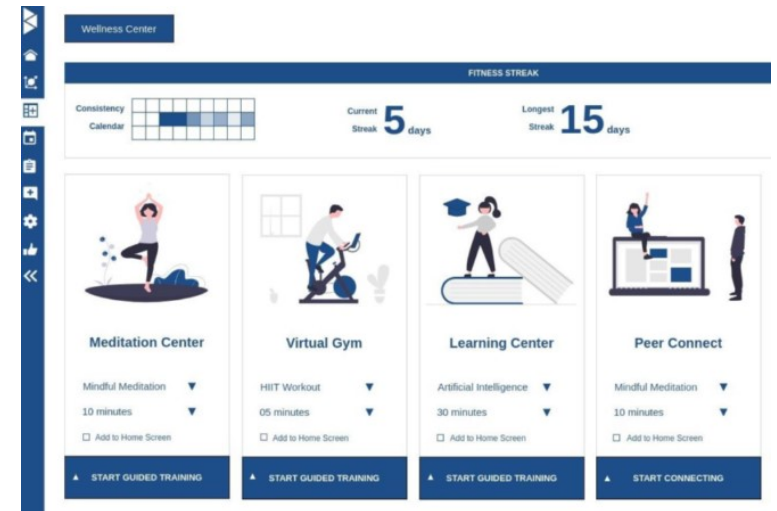

Fig. 6. Wellness Center

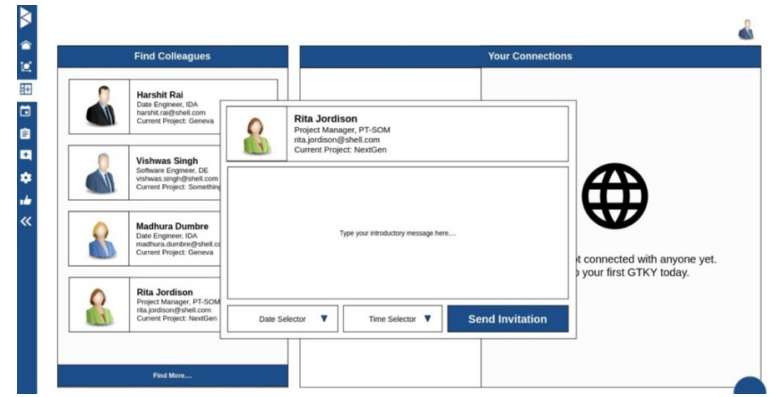

Fig. 7. Peer Connect

\subsubsection{Habit tracker}

It allows the user to keep a track of new habits he/she wants to inculcate. The user can select the target days for which he wants to follow the particular habit. Doing so ensures that the users are able to follow a healthy pattern in life.

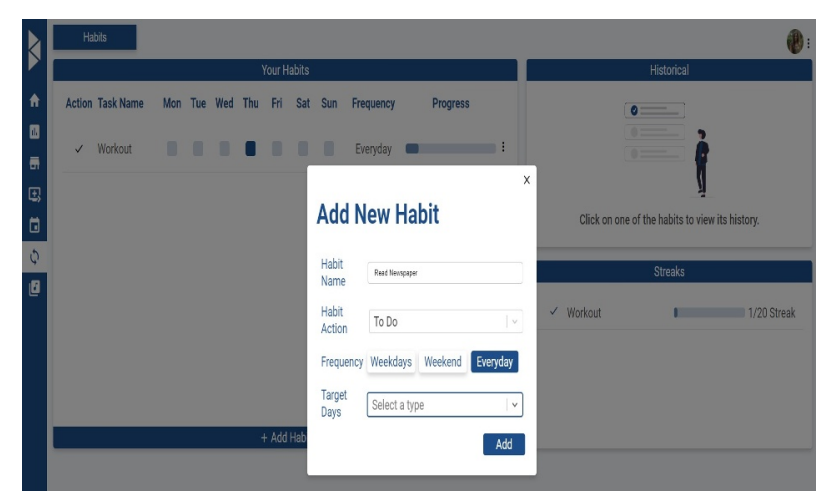

Fig. 8. Habit Tracker

\subsubsection{Scheduler}

The Scheduler is a Kanban board with a prioritization facility. Thus, the user can schedule and prioritize tasks he/she is supposed to perform. This will help our user to efficiently manage his tasks and be more organized. Which in turn will have positive mental health effects. 


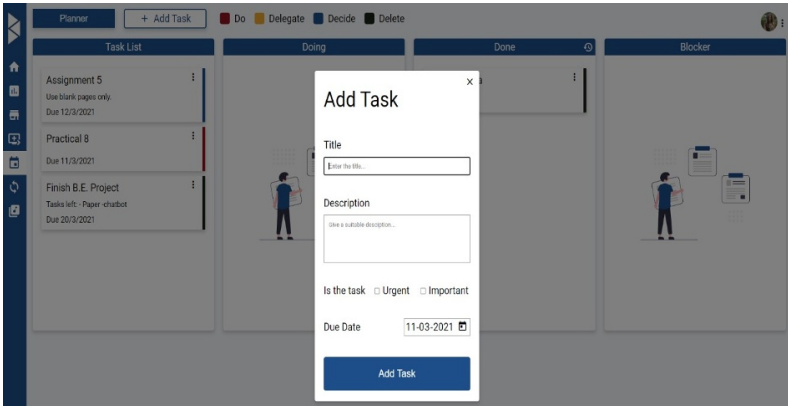

Fig. 9. Scheduler -1

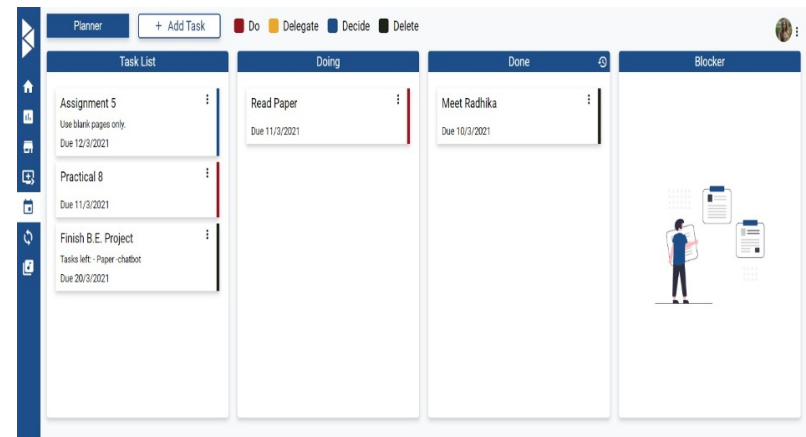

Fig. 10. Scheduler -2

\subsubsection{Seek Help}

In this section, the user can talk to and connect with professionals like psychiatrists, psychologist, counsellor, gym instructor, yoga instructor, anger management programs, etc. and thus get help from experts to deal with mental sickness.

\subsubsection{Chat-bot}

A chatbot is created using Dialogflow, to provide our user with an AI friend. Here, the user can talk to "Balancia," the virtual assistant. The virtual assistant, will give the user suggestions to deal with his/her situation.

\section{CONCLUSION AND SCOPE}

Mental health directly affects the way we think, feel and act. It also has a tremendous effect on our physical health. Unfortunately, recognising mental health concerns is a challenging undertaking, and mistake can lead to significant consequences. More so, many taboos have developed around mental sickness and thus people prefer avoiding the issue rather than consulting professionals. Hence, through this paper, we would like to help our community by developing a user-friendly android application which would allow them to easily diagnose their mental health problems and will also help and support them to improve their mental health, right from the user's home. It will also expand the reach and utilisation of e-mental health resources, particularly in rural/remote locations where there is a lack of awareness and availability of experienced specialists. This software will provide evidence-based services to meet current demands while also enhancing client trust in e-mental health care. By utilizing advances in technology, we aim to detect unhealthy digital behaviors by track changes and help users improve their digital lifestyle by providing them smart and personalized health recommendations.

The scope of the paper includes:

- Detecting any existing mental health issues.

- Detecting unhealthy digital behaviour

- Providing real-time in-app graphical visualization of user's habits.

- Helping users improve their digital and physical lifestyle by providing them with smart and personalized recommendations and in-app activities.

- Through the application, connecting the user with professionals.

- Keeping track of user's mental health condition

In conclusion, the aim is to develop a web application and android application to provide an end-to-end solution for the diagnosis of possibility of mental health illness and treating it.

\section{References}

[1]Understanding Mental Illness: https://www.nimh.nih.gov/health/statistics/mentalillness.shtml

[2] How India Sees Mental Health: https://www.weforum.org/agenda/2018/04/5charts-that-reveal-how-india-sees-mental-health/

[3] U. S. Reddy, A. V. Thota and A. Dharun, "Machine Learning Techniques for Stress Prediction in Working Employees," 2018 IEEE International Conference on Computational Intelligence and Computing Research (ICCIC), Madurai, India, 2018, pp. 1-4.

[4] M. P. Dooshima, E. N. Chidozie, B. J. Ademola, O. O. Sekoni, I. P. Adebayo, A Predictive Model for the Risk of Mental Illness in Nigeria Using Data Mining, International Journal of Immunology. Vol. 6, No. 1, 2018, pp. 5-16.

[5] M. Srividya, M. Subramaniam and B. Natarajan, "Behavioral Modeling for Mental Health using Machine Learning Algorithms" "Journal of Medical Systems" Vol. 42(5):88 May 2018.

[6] A. Kaur, "A hybrid approach of privacy preserving data mining using suppression and perturbation techniques," 2017 International Conference on Innovative Mechanisms for Industry Applications (ICIMIA), Bangalore, 2017, pp. 306-311.

[7] A. Kiran and D. Vasumathi, "A Comprehensive Survey on Privacy Preservation Algorithms in Data Mining," 2017 IEEE International Conference on Computational Intelligence and Computing Research (ICCIC), Coimbatore, 2017, pp. 1-7.

[8] C. Silva, M. Saraee and M. Saraee, "Data Science in Public Mental Health: A New Analytic Framework," 
2019 IEEE Symposium on Computers and Communications (ISCC), Barcelona, Spain, 2019, pp. 1123-1128, doi: 10.1109/ISCC47284.2019.8969723.

[9] K. Denecke, S. Vaaheesan and A. Arulnathan, "A Mental Health Chatbot for Regulating Emotions (SERMO) - Concept and Usability Test," in IEEE Transactions on Emerging Topics in Computing, doi: 10.1109/TETC.2020.2974478.
[10] A. Evfimievski. 2002. Randomization in privacy preserving data mining. SIGKDD Explor. Newsl. 4, 2 (December 2002), 43-48.

[11] Pyae, Aung. (2018). Study of Mental Health Data Science (Systematic Literature Review).

[12] M. Deshpande and V. Rao, "Depression detection using emotion artificial intelligence," 2017 International Conference on Intelligent Sustainable Systems (ICISS), Palladam, India, 2017, pp. 858862, doi: 10.1109/ISS1.2017.8389299. 